\title{
Efficient Hadronic Operators in Lattice Gauge Theory
}

\author{
UKQCD Collaboration presented by P. Lacock ${ }^{\mathrm{a}}$
}

${ }^{a}$ DAMTP, University of Liverpool, P.O. Box 169, Liverpool L69 3BX, Great Britain

\begin{abstract}
We propose the study of non-local gauge invariant operators to obtain an uncontaminated ground state for hadrons. The efficiency of the operators is shown by looking at the wave function of the first excited state, which has a node as a function of spatial extent of the operator.

Liverpool Preprint LTH-339 (Nov. 1994); hep-lat/9411013
\end{abstract}

\section{Introduction}

In quenched lattice gauge theory, hadronic states are created by acting with light quark (or anti-quark) creation operators on the vacuum. The ground state mass is then determined in the large (euclidean) time limit. In practice, however, only a limited range of $t$ is available, so methods are needed to create the hadronic ground state efficiently.

Since the main contamination of the ground state signal at larger $t$ values comes from the first excited state, one requires a hadronic creation operator which maximises the ground state relative to this first excited state.

One clear motivation for a trial hadronic operator comes from considering heavy quark hadrons: for heavy mesons $(c \bar{c}$ and $b \bar{b})$ the adiabatic approximation is well justified, so that they can be modelled as heavy point particles (quarks) bound by a central potential $V(R)$ between static colour sources. On a lattice efficient gluonic operators which create such a colour flux between static sources can be constructed using an iterative fuzzing algorithm [1]. This prescription creates gluonic fluxes with a dominant ground state and very few excited states. To extend this approach to lighter quarks, we use a lattice construction of a colour flux tube of length $R$ to join two light quarks in a gauge invariant manner. By varying $R$ we can explore the relative amplitude of ground state and excited state hadron created.

This relative amplitude is usually called the Bethe Saltpeter (BS) wave function of the hadron.

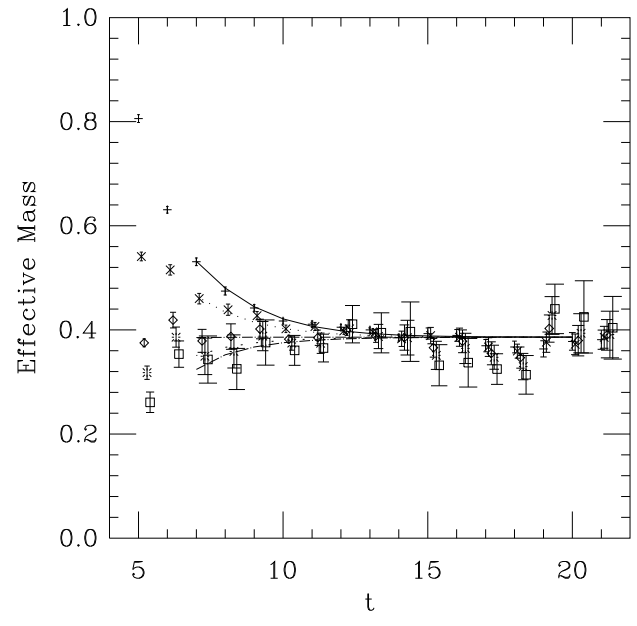

Figure 1. The effective mass for the $\rho$ (in lattice units) using the effective operators LL $(+)$, and $\mathrm{LF}$ with $\mathrm{R}=4(\times), 8(\diamond), 10(*)$ and $12(\square)$.

It is the overlap between a quark and antiquark at distance $R$ apart and the hadronic state which is an eigenstate of the hamiltonian (transfer matrix on a lattice). We use a fuzzed gluon flux prescription introduced in an earlier work with a similar approach [2] to join the quarks, which corresponds to the adiabatic wavefunction as defined by [3]. Some previous work has used quark and antiquarks in the (spatial) coulomb gauge instead [2,3]. This is less efficient (in our sense) and also leads to problems with image sources in the spatial periodic boundary conditions. 


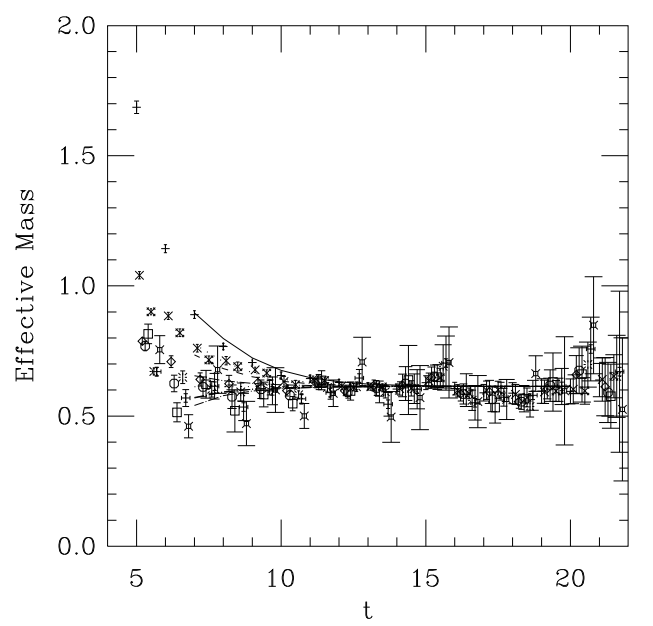

Figure 2. The effective mass for the Nucleon. Here we show the LLL, LLF and LFF operators for the various $R$ values (see text).

\section{Lattice Measurements}

We use light quark propagators in the $24^{3} \times 48$ configurations at $\beta=6.2$ and $K=0.14144$ using the clover action obtained by UKQCD [4]. Our most comprehensive wave function results come from an analysis of 12 configurations, although some quantities are available from a larger sample of 60 configurations. At these values of $\beta$ and $K$ the inverse lattice spacing is $a^{-1}=$ $2.73(5) \mathrm{GeV}$ (determined from the string tension), while $m_{\pi} / m_{\rho} \approx 0.77$.

We construct fuzzed gluon flux tubes following the successful methods used for studying the potential [1]:

$$
U_{n e w}=P_{S U(3)}\left(c U_{o l d}+\sum_{1}^{4} U_{u-b e n d s}\right) .
$$

Varying the parameters of the fuzzing prescription in our case gives relatively little change. Hence we select a smaller fuzzing level (5) and coarser fuzzing $(c=2)$ to minimise computer resources.

Since we have access only to light quark propagators from a single source $(\mathbf{0}, 0)$, we use a conventional local hadronic operator at the source. At the sink at time $t$, we use the spatially extended operator of length $R$ along a lattice axis. A sum over spatial positions (to have momentum zero) and a sum over all 6 orientations (to get correct $\left.J^{P C}\right)$ is used.

Here we follow the definitions and construction of the hadronic interpolating fields used by e.g. UKQCD [4]. The resulting hadronic correlation averaged over all configurations is then fitted to a two exponential function

$$
<h(0) H^{\dagger}(t, R)>=c_{0}(R) e^{-m_{0} t}+c_{1}(R) e^{-m_{1} t}
$$

We use a simultaneous fit to data at all $R$ values and $t$ values by making use of modified correlated $\chi^{2}$ fits, which models the correlations between dif-

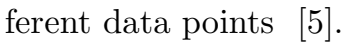

In the following we use $R=4,8,10$ and 12 , while the local operator corresponds to $R=0$. It will be shown below that this range of $R$ values is broad enough for the hadrons considered here. Statistical errors are determined using a bootstrap analysis.

For the mesons we form local-fuzzed (LF) correlations by replacing one local propagator in the usual (LL) formalism by the fuzzed propagator discussed above. The effective mass results for vector meson are shown in Fig. 1. There are five observables corresponding to LL, and LF with the four different $R$ values stated above.

For the nucleon, we consider two different nonlocal operators. These involve a di-quark separated from a quark (single fuzzed - LLF) and an arrangement of three quarks all separated (double fuzzed - LFF). Fig. 2 shows the results thus obtained for the nucleon.

Both figures clearly show that, by using fuzzed non-local operators, the plateau in the data starts at smaller values of $t$ than for the purely local observable. The fuzzed data have larger errors at lower $t$ (compared to the unfuzzed ones), but for larger $t$ the errors are comparable. The unfuzzed contribution thus has an accurate but irrelevant component.

The two exponential fit, which is stabilised by using several hadronic correlations to fit simultaneously, has the advantage that the ground state can be better isolated than by using a single exponential fit. 


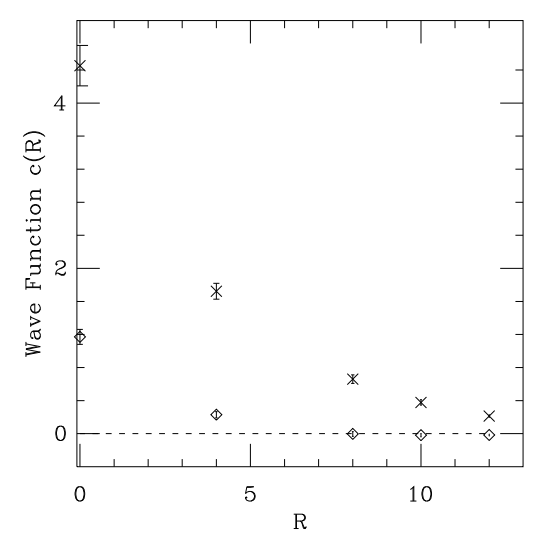

Figure 3. The $\pi$ wave function for the ground state $(\times)$ and first excited state $(\diamond)$, as functions of the length of the gluonic flux tube.

\section{Wave Functions}

To obtain a reasonably accurate determination of the wave function of the first excited state with our limited statistics, we fix the difference between the ground state and first excited state masses for the hadrons from results obtained recently by the UKQCD Collaboration [6]. These consist of hadronic propagators smeared at the source and sink (SS) and at the source only (SL) by applying the Jacobi smearing method on the existing 60 configurations at the origin, obtained on the $24^{3} \times 48$ lattice at $\beta=6.2$ with $K=0.14144$ 四. A big advantage of having SS, SL and LL operators is that it allows a factorising fit. These in turn provide tight constraints on the ground and excited state masses [6]. We then proceed as before, fitting the local and non-local quark propagators calculated from the subset of 12 configurations to the fit function given above.

The wave functions for the ground and first excited states obtained from the local operators and those involving only one fuzzed link, normalised at distance zero, are shown in Figs. 3 and 4 for the $\pi$ and Nucleon respectively. The wave functions for the $\rho$ and $\Delta$ show similar behaviour. The results for the ground state wave function are in agreement with those obtained previously in the literature using similar gauge invariant definitions

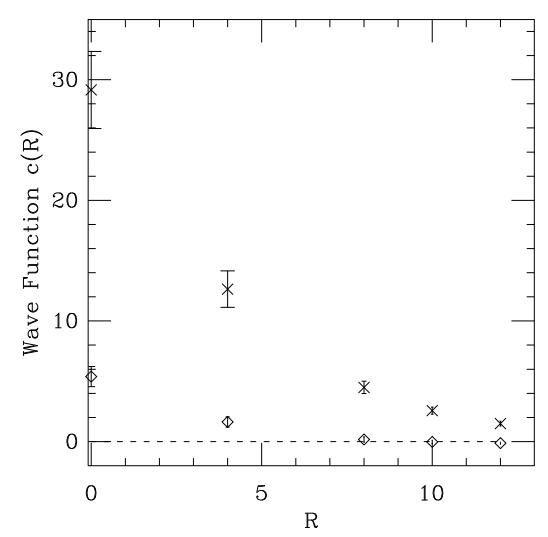

Figure 4. As in Fig. 3, but for the Nucleon, using the LLF effective operators.

of the wave function [2,3].

As far as we are aware, the excited state wave function has not been studied either for mesons or baryons. The interesting feature that can clearly be seen for all the hadronic observables considered here is the presence of a node in the excited state wave function as a function of the length of fuzzed links connecting the propagator ends at the sink, where the ratio of ground state wf to excited state wf becomes zero. This particular spatial extent $\left(R \approx 8\right.$ or $\approx 3 \mathrm{GeV}^{-1}$ in physical units), which is more or less the same for all the hadronic observables, thus seems to be an optimal choice for producing a clean ground state mass, since the contamination of the ground state by higher excited states has been minimised.

\section{REFERENCES}

1. S. J. Perantonis, A. Huntley and C. Michael, Nucl. Phys. B326 (1989) 544.

2. R. Gupta, D. Daniel and J. Grandy, Phys. Rev. D48 (1994) 3330.

3. K. B. Teo and J. W. Negele, Nucl. Phys. B (Proc. Suppl.) 34 (1994) 390.

4. UKQCD Collaboration, C. R. Allton et al., Phys Rev D49 (1994) 474.

5. C. Michael, Phys. Rev. D49 (1994) 2616;

C. Michael and A. McKerrell (in preparation)

6. UKQCD Collaboration, work in progress. 